\title{
Loss of Arterial Blood Volume Leads to Aortic Dissection
}

\author{
Ermoshkin Vladimir Ivanovich* \\ Physicist, Russian new University (RosNOU), Moscow, Russia
}

*Corresponding author: Ermoshkin Vladimir Ivanovich, Physicist, Russian new University (RosNOU), Moscow, Radio str, 22, Russia

ARTICLE INFO

Received: 幽 September 07, 2020

Published: 陆 September 22, 2020

Citation: Ermoshkin Vladimir Ivanovich. Loss of Arterial Blood Volume Leads to Aortic Dissection. Biomed J Sci \& Tech Res 30(4)-2020. BJSTR. MS.ID.004975.

\section{ABSTRACT}

\section{Annotation}

The problem of dissection and rupture of the aorta has not yet been solved. It is believed that this disease is caused by several risk factors: atherosclerosis, hypertension. Another risk factor is Smoking. And, of course, old age. This article provides a more plausible reason and mechanism for this event, but this is still a hypothesis.

\section{Goal}

It has been 9 years since the New Theory of CVD began to be created. About 40 articles on this topic have been published in English and Russian. The reaction of the leaders of Russian medicine is still zero. The New Theory develops on its own. The author of the theory believes that it is time to make some generalizations, discuss a New Theory and in particular the mechanism of sudden aortic injury.

\section{Method}

Study of numerous sources of information published on the Internet. Discussion of proposed ideas at conferences, publication of original articles in Russian and English-language medical journals.

\section{Results}

This hypothesis follows from the New Theory of CVD.On average, the heart pushes blood through the aorta 70 times a minute. We usually think of the aorta as a tube with elastic walls. Blood flows through this tube to all organs. The main function of the aorta is the distribution of blood with a pressure of 120-80 $(\mathrm{Hg})$ across various vascular pools. The diameter of the aorta and major arteries decreases in size on average with distance from the heart (considering the branching of blood vessels). The aorta itself is complete. Three lining of the aorta is histologically a whole. The inner layer of the aortic wall is called intima, the middle layer is called media, and the outer layer is called adventitia. The speed of blood flow in the aorta is high, up to $50 \mathrm{~cm} / \mathrm{sec}$. The pressure wave running from the heart along the aorta and arteries is called the pulse, its speed of propagation is up to $70-80 \mathrm{~cm} / \mathrm{sec}$. Thus, the aorta is an elastic tube up to $4 \mathrm{~cm}$ (near the heart) in diameter, which, depending on the specific blood pressure in a particular place, can change its internal and external diameters, of course, within certain limits from a certain minimum to a certain maximum.

At times when the arterial blood pressure (ABP) in a particular place of the vessel reaches a maximum, the internal and external diameter of the aorta reaches a maximum, and the thickness of the walls of the aorta at the same time - a minimum. At times when blood pressure reaches a minimum, the internal and external diameter reaches a minimum, and the wall thickness reaches a maximum. Thus, the walls of the aorta about 70 times a minute, then reduce their thickness, then increase.The lumen of the aorta or major artery cannot even in emergency cases decrease to zero, while maintaining a rounded shape, and it cannot increase to too large a value. The walls of the aorta and arteries have numerous small "vessels of vessels", they are necessary to maintain optimal performance of the multilayer structure. With maximum compression of the artery walls, capillary blood circulation worsens or is interrupted, and in other phases of the pulse wave, nutrition is restored. 


\section{a. Known Predictors of Aortic Dissection [1]}

At the histological level: there is a "necrosis", a lesion of the middle layer of the aorta. Doctors state "rarefaction" of the middle elastic layer. The middle layer becomes not dense enough, and the distance between the media fibers increases in the transverse direction relative to the axis of the vessel. It is based on a decrease in elasticity or organic, cellular changes in the structures of the aorta. The tissues soften and are easily degradable. Usually we are talking about congenital factors, in $20 \%$ of situations there is an acquired cause of the pathological process.

\section{b. Here is the Position of Official Medicine for the Reasons of Dissection $\backslash$ Rupture of the Aorta}

The first cause is a congenital degenerative lesion of connective tissue.Second reason: pressure overload of the aorta in hypertension, due to which there are violations in one of the membranes in the middle elastic shell. Next, there may be a separation of intima from the media, blood may get between the layers.The third cause is atherosclerosis, intramural hematomas, atheromatous plaques, infections, previous operations, injuries, ulcers near blood vessels. Jumps down and in General sharp changes in blood pressure can lead to aortic dissection - this is the opinion of medicine. But why? It will be shown below that the reason is the opening/closing of large anastomoses.The question. What is the specific mechanism of aortic dissection in a person who is healthy from birth? What forces cause the intima to peel off from the media? And why does this happen most often in the large aortic arch? There are no convincing answers.

\section{c. The position of the New Theory of CVD [2-16] on cause} and mechanism

The pathogenesis may be as follows. During frequent physical or psychological stress, arterial blood pressure (ABP) may increase. This is also facilitated by the sedentary lifestyle of modern people, hypodynamic. When ABP increases, shunts or arteriovenous anastomoses (AVA) [2-4] may open to protect the arteries from excessive stretching. Essentially, AVA are valves in the cardiovascular system that save us from high $\mathrm{ABP}$ and premature hemorrhagic strokes and heart attacks. During the opening of the AVA, part of the arterial blood is transfused into the veins, and the ABP drops. After the AVA closes, the pressure can rise again - this is how the notorious "jumps ABP". Over the years, at some points, the volume of arterial blood may decrease, and venous blood may increase. There is congestion, edema, varicose veins, ascites,overweight. This is also facilitated by the Earth's gravity. Let me remind you that in a healthy person, the volume of arterial blood is small, only $15 \%$. With frequent AVA openingsthe percentage of arterial blood volume to venous in humans decreases. The body can replenish blood volume, increasing the pumping of blood through the right ventricle, i.e. through the small circle of blood circulation, through the pulmonary vessels. Regulation of blood flow through the right and left ventricles is performed automatically, considering the Starling-Frank law and neurohumoral regulation. But with frequent discoveries of AVA, especially with hypodynamic, the mechanism of regulating the ratio of arterial and venous blood fails. Over time, significant venous fullness appears due to an increase in venous blood and intercellular fluid. Additionally, shortness of breath, cough, and asthma occur due to attempts to increase blood flow through the small circle and due to the formation of an incomplete left ventricular ejection fraction.

Gradually, the body is forced to adapt to the decreasing volume of arterial blood, i.e. to a volume of less than $15 \%$. This means that the internal volume of the arterial bed should be reduced, and this volume should always be filled with blood. Such phenomena are often recorded by doctors: spasm of small arteries and arterioles (a sign - cold hands and feet), narrowing of large arteries of the brain, heart, and legs. With a critical spasm of the arteries, strokes and heart attacks are possible.So, a significant decrease in the volume of arterial blood leads to a decrease in the internal volume of the arterial space (bed). A decrease in volume leads to transverse compression of the arteries and aorta by external forces. When sitting or standing, the gravity of the blood column in the vertical vessel as if "breaks" the intima from the media. In this case the elastic force of the elastic layer of the aorta and arteries prevent the diameter reduction of vessel they operate in the opposite direction. The middle elastic layer acts as a rubber tube and contributes to the increase of thevessel lumen to the optimum, i.e. to the average value. Strictly speaking, all these issues should be considered comprehensively, considering two positions: from the static side and from thedynamic side. In this article, the physical forces acting on the aorta are considered only from the static side. Dynamic effects are simply superimposed on static ones, but they are not covered in this article.

It is obvious that the uppermost parts of the aorta are subject to the greatest compression, because for this section the height of the blood column in the aorta is the maximum! It is on the large aortic arch that forces are formed with the greatest separation of intimacy from media! This is physics.External forces when blood leaks through the AVA compress the aorta (suction or a wave of negative blood pressure), affecting the entire layer of intima, especially in its upper part (the bend of the aorta), internal elastic forces (media and adventitia) act in the opposite direction, trying to hold the lumen and thereby expand (make it loose) the media layer. The line of the greatest pressure jump runs just between the layers of intimacy and media. That is why two transverse forces directed in different directions separate the aorta between the intima and the media, and sometimes break them. In this case, the media, which is in a stretched state, experiences micro-ruptures and lack of blood circulation of the micro vessels of the aorta. Lack of nutrition, transverse ruptures, and the inflammatory process can lead to fibrosis, to an increase in the hardness of the aorta and 
arteries. Note that official medicine indirectly confirms all these effects but does not explain the mechanism: there is a "rarefaction" and "media necrosis" of the middle elastic layer.

Note also that, apparently, the increase in the hardness of all the arteries, cardiomyopathy, the appearance of atrial fibrillation, atherosclerosis and plaque growth also mainly occur with the participation of the same cause, that is, due to AVA and due to the constant loss of arterial blood volume.Here is an example from physics. If you fill a bottle with water, then quickly turn it over and submerge the neck of the bottle (similar to capillaries and AVA) at least a couple of $\mathrm{cm}$ in a basin of water (venous pool), the water from the bottle will not be poured out. So that something spills out,it is necessary to compress the walls of the bottle (in other words, reduce the internal volume of the bottle). Assume mentally that the walls of the bottle are rubber, compressible. It immediately becomes clear that the reduction of the internal volume of the bottle will depend on the degree of elasticity of the rubber. In addition, in the upper part of the rubber bottle (analogous to bending the aorta), the effect of reducing the diameter of the vessel will be greater. But if the walls of the bottle are glass, i.e. incompressible, then there will be no water leaks from the bottle (in our case, arterial blood) at all!

Thanks to these findings, the mechanism of aortic dissection or rupture is clarified.Well, how to conduct prevention and how to save a person? Apparently, it is necessary to continuously monitor the loss of arterial blood in the human cardiovascular system and, in the case of some losses exceeding a given threshold, to make direct blood transfusions from veins to arteries, or to find effective physical exercises and procedures that allow this to be done. Of course, this is a difficult task.Another solution is to continuously monitor large AVA anastomoses, possibly creating artificial AVAs (parallel to large natural AVAs) with blood pumping in the opposite direction.Third solution. The solution to old: constant moderate physical activity helps to prolong life. Apparently, physical activity (especially light running, swimming, physical exercises) helps restore the balance of arterial and venous blood, promotes additional blood pumping in the small circle of blood circulation.I wonder if prominent medical scientists will find any reason to reject this hypothesis, too?Previously, they were able to do this. They have simply been silent for 9 years, while the New theory of CVD was developing.

\section{d. Note:}

As for the promising nitric oxide (NO) molecules for the treatment of arteries and aortic, it must be said that the hopes were only partially fulfilled. The molecule works well only under optimal conditions for pressure, wall tension, and other factors. Under the influence of damaging factors (namely, the missing volumes of arterial blood), the inner layer of endothelial vessels is destroyed. As a result, the vessels cannot produce nitric oxide and therefore narrow, the pressure increases. When blood moves through the vessels under high pressure, it further damages the endothelium.
The vessels narrow even more. The task of doctors is different: regularly replenish the arterial pool, this will be called prevention of atherosclerosis.

\section{Conclusions}

a) The emergence of this hypothesis for practical medicine is difficult to overestimate. Until July 2020, the cause and mechanism of aortic dissection and rupture were unknown. The true causes of atherosclerosis were also unknown. So, the author of the hypothesis believes. Now this hypothesis becomes known to everyone. It is necessary to periodically replenish the volume of arterial blood.

b) It is surprising that several "medical hypotheses" based on the ideas of the New theory of CVD were linked by invisible threads. An unknown link for medicine was the "invisible AVAanastomoses". Meanwhile, the development of the New Theory continues, it is still far from complete.

\section{References}

1. Internet resource. Russian.

2. (2007) Internet resource. Clinical case. Russian.

3. Lukyanchenko VA (2016) Cardiometric signs of performance of arteriovenous anastomosis in human cardiovascular system. Cardiometry (8): 22-25.

4. Ermoshkin VI (2016) New theory of arrhythmia. Conceptual substantiation of arrhythmia mechanisms. Cardiometry (8): 6-17.

5. Ermoshkin V (2017) The Cause of Some Cancers because of the Open Arteriovenous Anastomoses. J Gastrointest Cancer Stromal Tumor 2: 1000111.

6. Ermoshkin Vladimir Ivanovich (2019) New Theory of CVD/Cancer and Diabetes. EC Cardiology 7.1 (2020): 01-06.

7. Ermoshkin VI (2017) The new theory of heart failure. London.

8. Vladimir Ermoshkin (2018) Multiple Sclerosis. Why Did the Progressive Theory of Paulo Zamboni Not Find Support? But Then A New one Was Born! Int J clinical \& case 2(1): 04-06.

9. Ermoshkin VI (2016) Arteriovenous anastomoses and cardiovascular diseases. $8^{\text {th }}$ Cardiovascular Nursing \& Nurse Practitioners Meeting. Las Vegas, USA.

10. Ermoshkin VI (2016) Venous congestion due to large arteriovenous anastomoses. 566 Chiswick High Road, London, Greater London, W4 5YA, United Kingdom.

11. Ermoshkin VI (2016) Heart transplantation mysteriously eliminates arrhythmia. Cardiometry (8): 18-21.

12. Ermoshkin V (2016) Pathological Role of the Invisible Anastomoses J Bioengineer \& Biomedical Sci 6(5): 209.

13. Vladimir Ermoshkin (2017) The pathological mechanism of systemic inflammation in humans has opened. The reason is uncontrolled leakage of arterial blood through arteriovenous anastomoses. Int J Car \& Hear Heal 1(2): 12-14.

14. Ermoshkin VI (2020) Information about a single mechanism of many CVDs requires consideration at the top. Med links Cardiology.

15. Ermoshkin VI (2020) Cause and Mechanism of Aortic Dissection. Med links Cardiology.

16. (2020) The mechanism of pathological spasms of the arteries of the human brain. Med links Alternative medicine. 
ISSN: 2574-1241

DOI: $10.26717 /$ BJSTR.2020.30.004975

Ermoshkin Vladimir Ivanovich. Biomed J Sci \& Tech Res

(CC) (i) This work is licensed under Creative Commons Attribution 4.0 License

Submission Link: https://biomedres.us/submit-manuscript.php

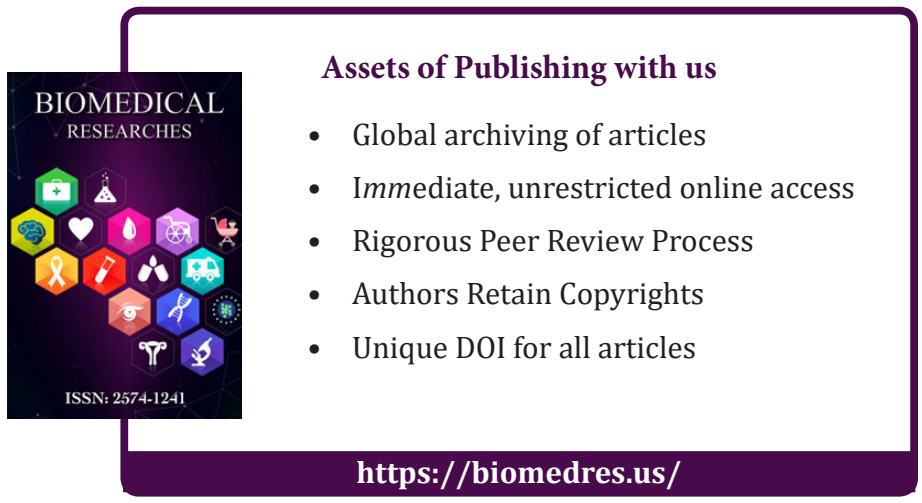

